\title{
LEF1-AS1 accelerates tumorigenesis in glioma by sponging miR-489-3p to enhance HIGD1A
}

\author{
Zhihua Cheng', Guangyu Wang ${ }^{1}$, Weiyi Zhu², Cong Luo ${ }^{1}$ and Zhilin Guo ${ }^{1}$
}

\begin{abstract}
Long non-coding (InCRNA) lymphoid enhancer-binding factor 1 antisense RNA 1 (LEF1-AS1) has been validated to be implicated in manifold cancers, whereas its function in glioma has not been understood thoroughly. Hence, in this study, we tested that LEF1-AS1 expression was significantly upregulated in glioma tissues and cell lines. Besides, knockdown of LEF1-AS1 repressed cell proliferation while activated apoptosis in glioma cells in vitro, and also suppressed tumor growth in vivo. RNA pull-down and luciferase reporter assays affirmed that LEF1-AS1 could bind with miR-489-3p. In addition, miR-489-3p expression was downregulated in glioma cells. Moreover, miR-489-3p depletion partly offset LEF1-AS1 knockdown-mediated function on proliferation and apoptosis. Further, HIGD1A identified as the target gene of miR-489-3p was upregulated in glioma cells. HIGD1A silence could restrict the process of glioma. In rescue assays, upregulation of HIGD1A remedied the inhibitory impacts of LEF1-AS1 silence on glioma cell growth. In summary, our studies corroborated the regulatory mechanism of LEF1-AS1/miR-489-3p/HIGD1A axis in glioma, suggesting that targeting LEF1-AS1 might be a promising method for glioma therapy in the future.
\end{abstract}

\section{Introduction}

Glioma is a common brain tumor in adults, accounting for about $60 \%{ }^{1}$. The distinct symbols of high-grade glioma contain neurocognitive impairment, poor quality of life as well as loss of life independence ability ${ }^{2}$. Although surgery has achieved great achievements in combination with radiotherapy and medication, the recurrence rate of glioma patients is still increasing ${ }^{3,4}$. Hence, it is essential to have a clear understanding of glioma and discover a brand-new target for treatment of glioma.

A number of literatures supported that long non-coding RNAs (lncRNAs) play an important role in the occurrence and development of various cancers, including glioma. For example, serum lncRNA HOTAIR serves as a new biomarker for the diagnosis and prognosis of glioblastoma

\footnotetext{
Correspondence: Zhilin Guo (guozhilin6502@163.com)

'Department of Neurosurgery, Shanghai Ninth People's Hospital, Shanghai Jiao Tong University, School of Medicine, No. 639 Zhizaoju Road, 200011 Shanghai, China

2Department of Neurosurgery, Shanghai Jing'an District Central Hospital,

No. 259 Xikang Road, 200040 Shanghai, China

These authors contributed equally: Zhihua Cheng, Guangyu Wang

Edited by: N Barlev
}

multiforme ${ }^{5}$. LncRNA NEAT1 knockdown represses migration and invasion of glioma cells through regulating SOX2 targeted by miR- $132^{6}$. Silencing lncRNA MIR22HG suppresses glioblastoma progression via inhibition of Wnt $/ \beta$-catenin signaling ${ }^{7}$. The oncogenic role of LEF1AS1 has been studied in prostate cancer and myeloid malignancy previously ${ }^{8,9}$. More importantly, LEF1-AS1 was abnormally upregulated in glioma tissues and closely associated with unsatisfactory prognosis of glioma patients via public database. Meanwhile, present research also confirmed that LEF1-AS1 was remarkably upregulated in tissues from glioma patient. However, the function of LEF1-AS1 in glioma has not been elucidated yet.

Emerging studies have highlighted the importance of competing endogenous RNA (ceRNA) network in multiple cancers. In this mechanism, lncRNAs functioned as sponges of microRNAs (miRNAs) to liberate mRNAs so that they could be released to code into proteins ${ }^{10}$. LncRNA NEAT1 and linc00152 exerted tumor-promoter role in glioma via modulating ceRNA network ${ }^{6,11}$. Therefore, we intended to investigate that whether LEF1-AS1 also played a role in glioma through the ceRNA network. 
MiRNAs are a group of non-coding RNAs with 18-25 nucleotides in length, but without the protein-coding capacity $^{12}$. MiRNAs are ubiquitous in almost all biological processes, including morphogenesis of metastasis, differentiation, and proliferation ${ }^{13,14}$. For instance, miR-103a-3p suppresses glioma cell proliferation and apoptosis ${ }^{11}$. MiR124 signaling pathway affects cell proliferation, invasion and metastasis in glioblastoma multiforme ${ }^{15}$. MiR-423-5p is beneficial for enhancing the malignant phenotypes in glioblastomas ${ }^{16}$. In the present study, LEF1-AS1 expression mainly distributed in cytoplasm, indicating the posttranscriptional regulation potential of LEF1-AS1 in glioma. Further, bioinformatics suggested the binding relation between LEF1-AS1 and miR-498-3p, which was confirmed by the mechanical experiments. Nevertheless, the role of miR-498-3p in glioma was uncovered.

Moreover, the decisive role of protein-coding genes in cancer induced us to find out the target gene of miR-498$3 p$ in glioma cells. Due to the most obviously regulatory effect of miR-498-3p overexpression on HIGD1A expression, HIGD1A was chosen as our researching focus. Therefore, whether LEF1-AS1 could regulate HIGD1A via miR-498-3p to affect glioma development was worthy exploring.

Overall, the main task of our study was to investigate the role of LEF1-AS1 in glioma. Besides, the regulatory mechanism of ceRNA network consisting of LEF1-AS1, miR-498-3p and HIGD1A was elucidated in glioma.

\section{Materials and methods Tissue samples}

The matched glioma tissues and non-tumor tissues were collected from 46 glioma patients, with the approval from the Ethics Committee of Shanghai Ninth People's Hospital and the informed consents from all patients. The participants didn't receive chemotherapy or radiotherapy prior to surgery. Following frozen by liquid nitrogen immediately after surgical resection, tissue samples were kept at $-80^{\circ} \mathrm{C}$ for further analysis.

\section{Cell lines}

Human glioma cell lines, including U251 (astrocytoma), T98MG (glioblastoma), SWO38 and U373MG (astrocytoma), and control cell line (HEB and NHA), provided by ATCC (Manassas, VA), were propagated in the DMEM (Invitrogen, Carlsbad, CA) under $37^{\circ} \mathrm{C}$ with $5 \% \mathrm{CO}_{2}$. The $10 \%$ FBS (Gibco, Waltham, MA) and 1\% Pen/Strep solution were applied for supplementing DMEM.

\section{Quantitative real-time PCR (qRT-PCR)}

Total RNAs extracted by TRIzol (Invitrogen) from U251 and T98MG cells were used for cDNA synthesis with the help of Reverse Transcription Kit (Toyobo, Osaka, Japan). SYBR Green Super Mix (Bio-Rad, Hercules, CA) was applied for qPCR experiment. Data were standardized to GAPDH or U6, and relative expression was calculated by $2^{-\Delta \Delta C T}$ method.

\section{Transfection}

The shRNAs of LEF1-AS1, HIGD1A and the relative negative control (NC) sh-NC, the miR-489-3p mimics/ inhibitor and $\mathrm{NC}$ mimics/inhibitor, together with the pcDNA3.1/LEF1-AS1, pcDNA3.1/HIGD1A and NC pcDNA3.1, were procured from Genepharma (Shanghai, China) for transfection (2 $\mu \mathrm{g} /$ well). Lipofectamine 2000 (Invitrogen) was used for transfection of U251 and T98MG cells with indicated plasmids for $48 \mathrm{~h}$.

\section{Colony formation assay}

Transfected glioma cells were planted in triplicate in the 6 -well plates (500 cells/well) for the 14 days of incubation at $37^{\circ} \mathrm{C}$. The colonies with more than 50 cells were fixed for $30 \mathrm{~min}$ in $4 \%$ formaldehyde (Sigma-Aldrich) and sequentially stained for $5 \mathrm{~min}$ by using $0.5 \%$ crystal violet (Sigma-Aldrich), followed by counting manually.

\section{EdU assay}

EdU assay was performed in U251 and T98MG cells following the user guidebook of Cell-light ${ }^{\mathrm{TM}} \mathrm{EdU}$ ApolloR567 in Vitro Imaging Kit (Ribobio, Guangzhou, China). Upon addition of fresh medium, EdU was added and cells were incubated for $2 \mathrm{~h}$. After incubation, cells were washed by PBS to remove the medium containing free EdU. Next, cells were immobilized for $30 \mathrm{~min}$ in $4 \%$ paraformaldehyde (Sigma-Aldrich) before being stained by DAPI (Sigma-Aldrich), and then observed under fluorescent microscope.

\section{Flow cytometry for apoptosis}

Annexin V apoptosis detection kit (Life technologies, Grand Island, NY) was employed for flow cytometry analysis. $2 \times 10^{5}$ fresh glioma cells were reaped and put on ice for $1 \mathrm{~h}$. Then, the resuspended cells in $100 \mu \mathrm{l}$ binding buffer (Nanjing KeyGen Biotech Co., Ltd.) were processed with $5 \mu \mathrm{l} \mathrm{PI}(100 \mu \mathrm{g} / \mathrm{ml})$ and $1 \mathrm{U} / \mathrm{ml}$ ribonuclease in a dark environment at room temperature for half an hour. Afterwards, cells were cultivated with $5 \mu \mathrm{l}$ of Annexin VFITC for additional 15 minutes, based on the manufacturer's instructions. Subsequently, FACSCalibur (BD Biosciences, San Jose, CA) was utilized to analyze cells with an Attune flow cytometer (Thermo Fisher Scientific, Inc.). Software FlowJo 1.1.0 (Tree Star, Inc.) was used to determine the apoptosis rate.

\section{Terminal dexynucleotidyl transferase (TdT)-mediated dUTP nick end labeling (TUNEL) assay}

Transfected glioma cells were subjected to treatment with $4 \%$ paraformaldehyde for $15 \mathrm{~min}$ and $0.25 \%$ Triton-X 
100 for $20 \mathrm{~min}$. After that, cells were treated with TUNEL detection kit (Roche, Basel, Switzerland). Following DAPI dying, cells were analyzed under fluorescent microscope.

\section{Western blot}

Total protein samples were subjected to electrophoresis on the 10\% SDS-PAGE and then shifted onto the PVDF membranes. After sealing with the $5 \%$ skimmed dried milk, membranes were cultivated with primary antibodies against control GAPDH (ab8245; 1:1000), Bcl-2 (ab32124; 1:1000), Bax (ab32503; 1:1000), Total caspase-3 (ab13847; 1:500), Cleaved caspase-3 (ab49822; 1:500), and the appropriate HRP-tagged secondary antibodies (all, Abcam, Cambridge, MA). Protein signals were monitored by the detection system of enhanced chemiluminescence (ECL; Santa Cruz Biotechnology, Santa Cruz, CA, United States).

\section{Fluorescence in situ hybridization (FISH)}

The RNA FISH probe designed for LEF1-AS1 was acquired from RiboBio and employed as per the standard method. After staining cells with Hoechst, images were captured by use of confocal microscope (OLYMPUS, Tokyo, Japan).

\section{RNA pull-down assay}

The wild-type (WT) or mutated (Mut) sequences of miR-489-3p containing the putative LEF1-AS1 binding sites were biotinylated into Bio-miR-489-3p-Wt/Mut, which were further subjected to incubation with the cell lysates for $1 \mathrm{~h}$. Finally, the beads were added to collect pull-downs and qRT-PCR analysis of indicated RNAs was followed.

\section{Dual-luciferase reporter analyses}

The WT and Mut miR-489-3p binding sites within the LEF1-AS1 sequence or HIGD1A 3'-UTR were synthesized for inserting into the downstream of pmirGLO vectors (Promega, Madison, WI). The LEF1-AS1-WT/Mut and HIGD1A-WT/Mut were formed and co-transfected with indicated plasmids for $48 \mathrm{~h}$, followed by the detection of luciferase activity via Dual Luciferase Assay System (Promega).

\section{RNA immunoprecipitation (RIP) assay}

$1 \times 10^{7}$ glioma cells lysed in the RIP lysis buffer were prepared for the immunoprecipitation with beads conjugated to antibodies (Millipore, Billerica, MA) against control IgG or human Ago2. Precipitated RNAs were assayed by qRT-PCR.

\section{In situ hybridization (ISH) staining}

ISH assay was performed to detect LEF1-AS1 expression in tissue samples. The experiment was conducted using the sensitive enhanced in situ hybridization kit of BOSTER Company (Wuhan, China) as required by supplier. The semi-quantitative ISH scoring standard was used to record the staining intensity and the number of positive zones. 4 grades, including 0 (negative), $1(<10 \%$ positive), 2 (10-50\% positive) and 3 ( $>50 \%$ positive), were used according to the staining proportion and intensity. The final scores were presented as low expression (0-1) and high expression (2-3).

\section{Xenograft model}

Male BALB/c nude mice (6-weeks; weight: $20-25 \mathrm{~g}$ ) were used for subcutaneous xenograft tumor experiment, with the approval of the Ethics Committee of Shanghai Ninth People's Hospital. $1 \times 10^{8}$ transfected cells were subcutaneously injected into nude mice, and tumor volumes were monitored every 4 days. Following 28-day of injection, mice were killed and tumors were carefully excised for weigh assessment.

\section{Hematoxylin and Eosin (H\&E) staining}

The collected tissues from xenograft model were fixed with $4 \%$ paraformaldehyde at $4{ }^{\circ} \mathrm{C}$, embedded in paraffin and then cut into $4 \mu \mathrm{m}$ sections. After de-paraffin, sections were rehydrated and stained with H\&E (SigmaAldrich) at $4{ }^{\circ} \mathrm{C}$ for $10 \mathrm{~min}$, finally observed under Olympus light microscope (magnification, $\times 200$ ).

\section{Immunohistochemistry (IHC)}

The tissue samples acquired in xenograft tumor assay were fixed and embedded in paraffin. After cutting, the $4 \mu \mathrm{m}$ sections were subjected to IHC experiment using anti-Ki67 antibody (ab15580; 1:100; Abcam) in line with the standard protocol. After washing with PBS, the sections were incubated with HRP-conjugated secondary antibody (Abcam), and examined under light microscope (Olympus; magnification, $\times 200$ ).

\section{Bioinformatics tools}

The high expression of LEF1-AS1 in glioma tissues and prognostic significance of LEF1-AS1 for glioma patients were analyzed by GEPIA (http://gepia.cancer-pku.cn/) online public database. StarBase website (http://starbase. sysu.edu.cn/index.php) was utilized to predict the potential downstream miRNAs of LEF1-AS1 and the potential downstream target genes of miR-489-3p.

\section{Statistical analysis}

Data analysis was developed by $t$ test or one-way analysis of variance (ANOVA) using GraphPad Prism 6.0 (La Jolla, CA), with $p<0.05$ as cutoff of statistical significance. Continuous variables of 3 or more independent assays were shown as the mean \pm SD. 


\section{Results}

LEF1-AS1 enhances the malignant growth of glioma cells

The previous study revealed that LEF1-AS1 was an oncogene in lung cancer. According to GEPIA public database, LEF1-AS1 was an aberrantly upregulated lncRNA in GBM tissues $(N=163)$ compared with normal tissues $(N=207)$ (Fig. S1a). Based on TCGA-GBM datasets, we found that high expression of LEF1-AS1 was closely related to short survival time of GBM patients (Fig. S1b), further indicating the potential contribution of LEF1-AS1 to the malignancy of glioma. Based on these results, we launched following investigations to probe into the precise role of LEF1-AS1 in glioma. Therefore, the expression pattern of LEF1-AS1 in glioma was measured via $\mathrm{qRT}$-PCR firstly. Data delineated that LEF1-AS1 was obviously upregulated in glioma tissues and cell lines (U251, T98MG, SWO38 and U373MG) compared with non-tumor tissue groups and control cell lines (HEB and NHA), respectively (Fig. S1c and 1a). Besides, ISH assay further confirmed that LEF1-AS1 was highly expressed in glioma tissue samples compared with non-tumor tissue samples (Fig. S1d). Moreover, we unveiled that glioma samples from patients at high grades (WHO3-WHO4 grades) expressed higher LEF1-AS1 than those from patients at less malignant grades (WHO1-WHO2 grades) (Fig. S1e). To ascertain the biological function of LEF1AS1 in glioma, we silenced LEF1-AS1 by sh-LEF1-AS1\#1/ 2 transfection in U251 and T98MG cells, which contained the highest level of LEF1-AS1 among the indicated glioma cell lines (Fig. 1b). The colony formation assay revealed that LEF1-AS1 silence remarkably decreased glioma cell proliferation (Fig. 1c). Similarly, the proportion of positive EdU stained cells in sh-LEF1-AS1\#1/2 transfected group was evidently less than that in sh-NC group (Fig. 1d). On the contrary, results of flow cytometry analysis exhibited an enhancement of glioma apoptosis in response to LEF1AS1 depletion (Fig. 1e). TUNEL assay indicated that knockdown of LEF1-AS1 potently accelerated cell apoptosis in glioma as well (Fig. 1f). Consistently, suppression of LEF1-AS1 resulted in an increase of cleaved caspase-3 and Bax while a decrease of Bcl-2 (Figs. $1 \mathrm{~g}$ and S1e). Thereafter, we also performed in vivo experiments through injecting sh-LEF1-AS1\#1 transfected glioma cells into mice. As anticipated, tumor derived from LEF1-AS1depleted cells looked smaller in size, along with a slower growth rate, than tumors originated from control cells (Fig. S2a). Also, we found the smaller volume and lighter weight of tumors with inhibited LEF1-AS1 than that of those in control group (Fig. S2b). Further, IHC staining assays elucidated that decreased $\mathrm{Ki} 67$ expression was observed in tumors with downregulated LEF1-AS1 (Fig. S2c). In brief, LEF1-AS1 downregulation conspicuously hindered glioma malignant growth both in vitro and in vivo.

\section{LEF1-AS1 sponges miR-498-3p in glioma cells}

To investigate the regulatory mechanism of LEF1-AS1 in glioma, we firstly implemented FISH assay to detect LEF1-AS1 localization. Results confirmed that LEF1-AS1 was mainly distributed in cytoplasm of glioma cells (Fig. 2a). Hence, we guessed that LEF1-AS1 played its role via ceRNA pattern. Bioinformatics data obtained from starBase provided the binding site between LEF1-AS1 and miR-498-3p (Fig. 2b). Next, results of qRT-PCR unveiled that miR-498-3p expression was downregulated in glioma cell lines in comparison with normal controls (Fig. 2c). Thereafter, qRT-PCR unveiled the elevated expression of miR-489-3p in LEF1-AS1-silenced U251 and T98MG cells (Fig. 2d). Afterwards, RNA pull-down assay demonstrated that LEF1-AS1 was highly enriched in BiomiR-489-3p-WT group not in Bio-miR-489-3p-Mut group (Fig. 2e). qRT-PCR assay illustrated that miR-489$3 p$ expression was increased by transfecting miR-489-3p mimics into glioma cells (Fig. 2f). Subsequently, we observed that the luciferase activity of LEF1-AS1-WT was suppressed by miR-489-3p mimics while that of LEF1AS1-Mut presented no notable changes (Fig. 2g). Altogether, LEF1-AS1 sponged miR-489-3p in glioma cells.

\section{MiR-498-3p depletion reversed the effects of LEF1-AS1 knockdown in glioma cells}

Through rescue experiments, we investigated whether LEF1-AS1 affected glioma cell proliferation and apoptosis via modulating miR-489-3p. MiR-489-3p expression was suppressed by miR-489-3p inhibitor in glioma cells (Fig. 3a). In addition, miR-489-3p expression was upregulated in sh-LEF1-AS1\#1/\#2 transfected cells, and then was normalized in cells co-transfected with sh-LEF1AS1\#1 and miR-489-3p inhibitor (Fig. 3b). In colony formation assay, we discovered that miR-489-3p depletion could offset the repressive influence of LEF1-AS1 downregulation on cell proliferation (Fig. 3c). Similarly, consequences from EdU experiments further verified the rescuing effects of miR-489-3p downregulation on LEF1AS1 depletion-hindered cell proliferation (Fig. 3d). Additionally, the elevated cell apoptosis induced by LEF1AS1 silence was recovered by miR-449b-5p inhibition, demonstrated by flow cytometry and TUNEL assays (Fig. 3e, f). Besides, the effects of LEF1-AS1 knockdown on cleaved caspase-3, Bax and $\mathrm{Bcl}-2$ proteins were countervailed by miR-489-3p suppression (Figs. $3 g$ and S3a). To sum up, LEF1-AS1 accelerated the progression of glioma via targeting miR-489-3p.

\section{HIGD1A silence curbed the malignant behaviors of glioma cells}

Though searching starBase (http://starbase.sysu.edu.cn), FLNB, ETS2, and HIGD1A were screened out as the potential downstream target genes of miR-498-3p under 


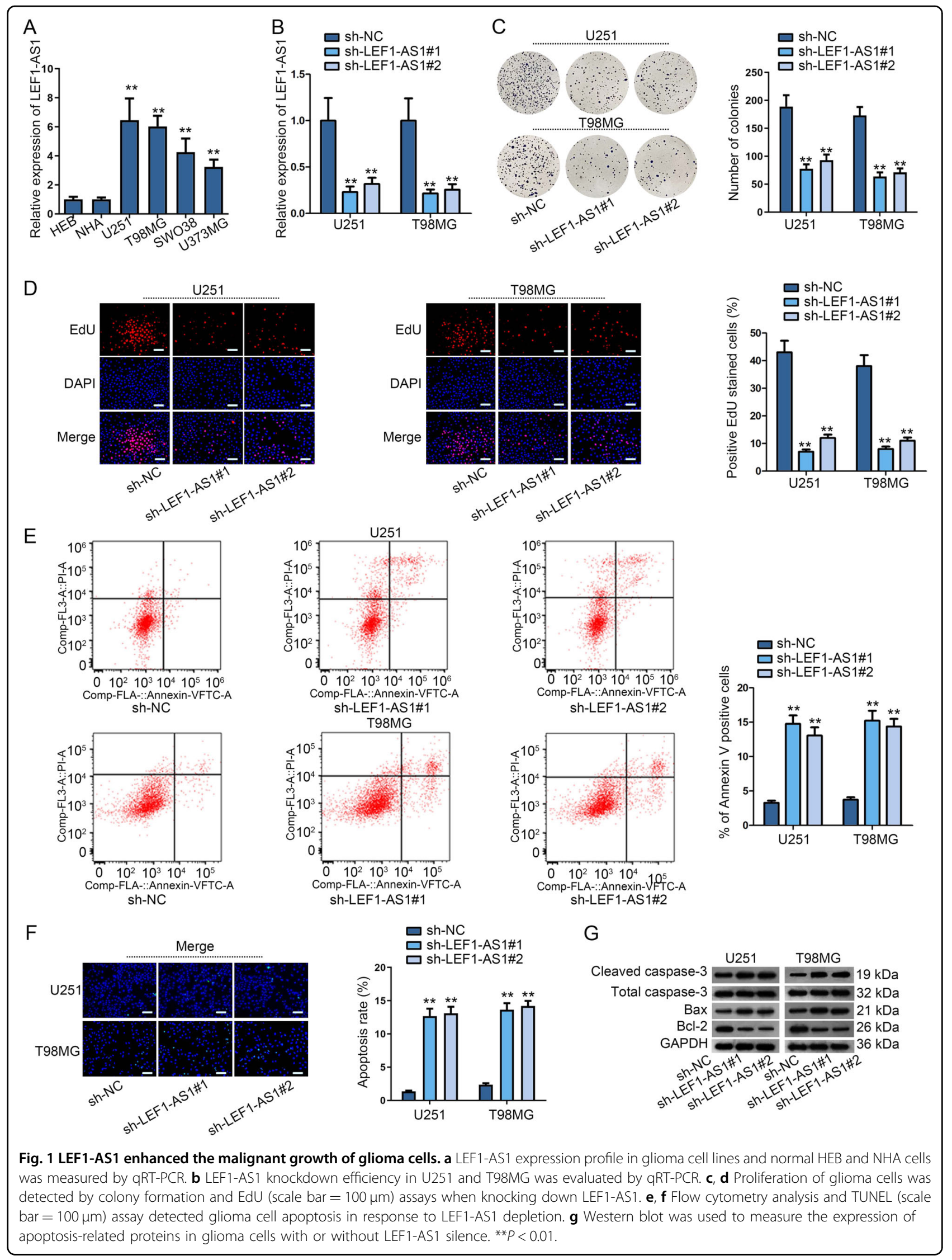


A

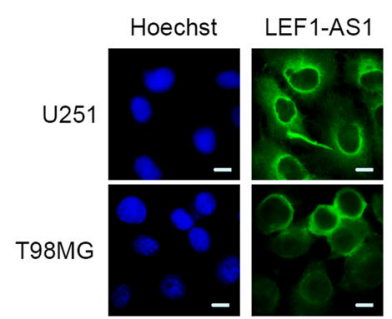

C

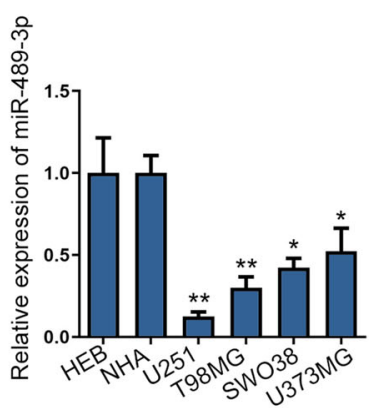

$\mathrm{F}$

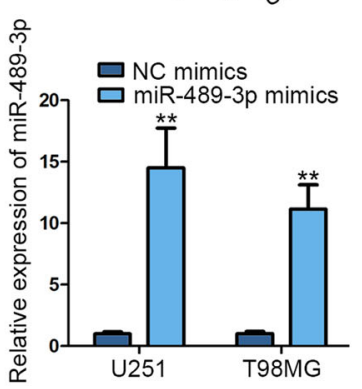

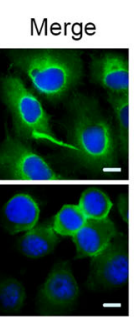

B

LEF1-AS1-WT: 5' accaagGAAGACUUGAUGUCAg 3'

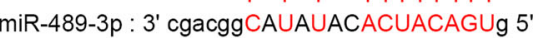

LEF1-AS1-Mut: 5' accaagCAUGUCUACUACAGUg 3'

E

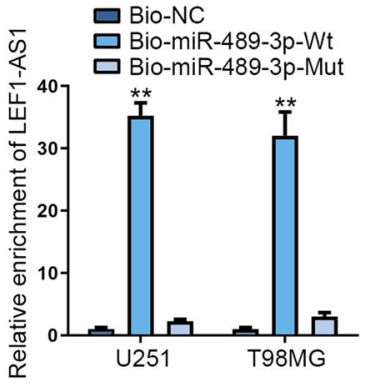

$\mathrm{G}$

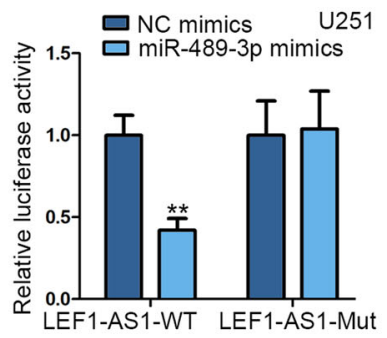

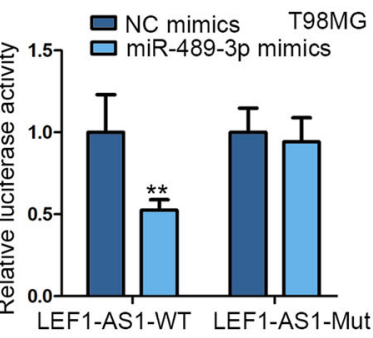

Fig. 2 LEF1-AS1 sponged miR-498-3p in glioma cells. a Cytoplasmic location of LEF1-AS1 in glioma cells was detected by FISH assay (scale bar = $10 \mu \mathrm{m}$ ). b Bioinformatics (starBase) presentation of binding sites between LEF1-AS1 and miR-489-3p. c MiR-489-3p expression was detected by qRTPCR in glioma cell lines and control cell lines. $\mathbf{d}$ MiR-489-3p expression was measured by qRT-PCR when downregulating LEF1-AS1. e RNA pull-down assay examined the interaction between LEF1-AS1 and miR-489-3p in glioma cells. $\mathbf{f}$ MiR-489-3p upregulation efficiency was examined by qRT-PCR in U251 and T98MG cells. $\mathbf{g}$ The luciferase activity of LEF1-AS1-WT/Mut was measured by luciferase reporter assay in NC mimics or miR-489-3p mimics transfected cells. ${ }^{*} P<0.05,{ }^{*} P<0.01$.

the condition of strict stringency and high stringency. Then, the expressions of these genes were detected in glioma cells when overexpressing miR-498-3p. Prominently, HIGD1A expression was suppressed by miR-4893p mimics while no obvious changes in FLNB and ETS2 expressions (Fig. 4a). Simultaneously, the complementary binding sites between miR-489-3p and HIGD1A were show in Fig. 4b. The expression of HIGD1A was tested in glioma cell lines (U251, T98MG, SWO38, and U373MG) and control cell lines (HEB and NHA). Results indicated that HIGD1A was highly expressed in glioma cell lines (Fig. 4c). Data of RIP assays disclosed that LEF1-AS1, miR-489-3p and HIGD1A were abundant in complexes precipitated by Ago2 antibody but not in compounds captured by IgG antibody (Fig. 4d). Later, it was revealed by luciferase reporter assay that miR-489-3p upregulation reduced the luciferase activity of HIGD1A-WT not that of HIGD1A-Mut, while such effect was then offset by upregulated LEF1-AS1 (Fig. 4e). Then, we examined the function of HIGD1A in glioma cells after validating the declined HIGD1A expression by sh-HIGD1A (Fig. 4f). It was revealed that HIGD1A depletion inhibited the proliferation and promoted the apoptosis of glioma cells (Fig. 4g-j). Meanwhile, HIGD1A knockdown inhibited the anti-apoptosis protein (Bcl-2) expression and stimulated pro-apoptosis protein (cleaved caspase- 3 and Bax) levels (Figs. 4k and S3b). In a word, HIGD1A silence curbed the malignancy in glioma.

\section{LEF1-AS1 fostered the development of glioma via enhancing HIGD1A expression}

Rescue assays were constructed to validate whether HIGD1A affected the modulatory effects of LEF1-AS1 on glioma cellular processes. To begin with, we confirmed the success overexpression of HIGD1A in cells transfected with pcDNA3.1/HIGD1A compared to those with pcDNA3.1 control (Fig. 5a). Colony formation and EdU assays testified that upregulating HIGD1A counteracted 

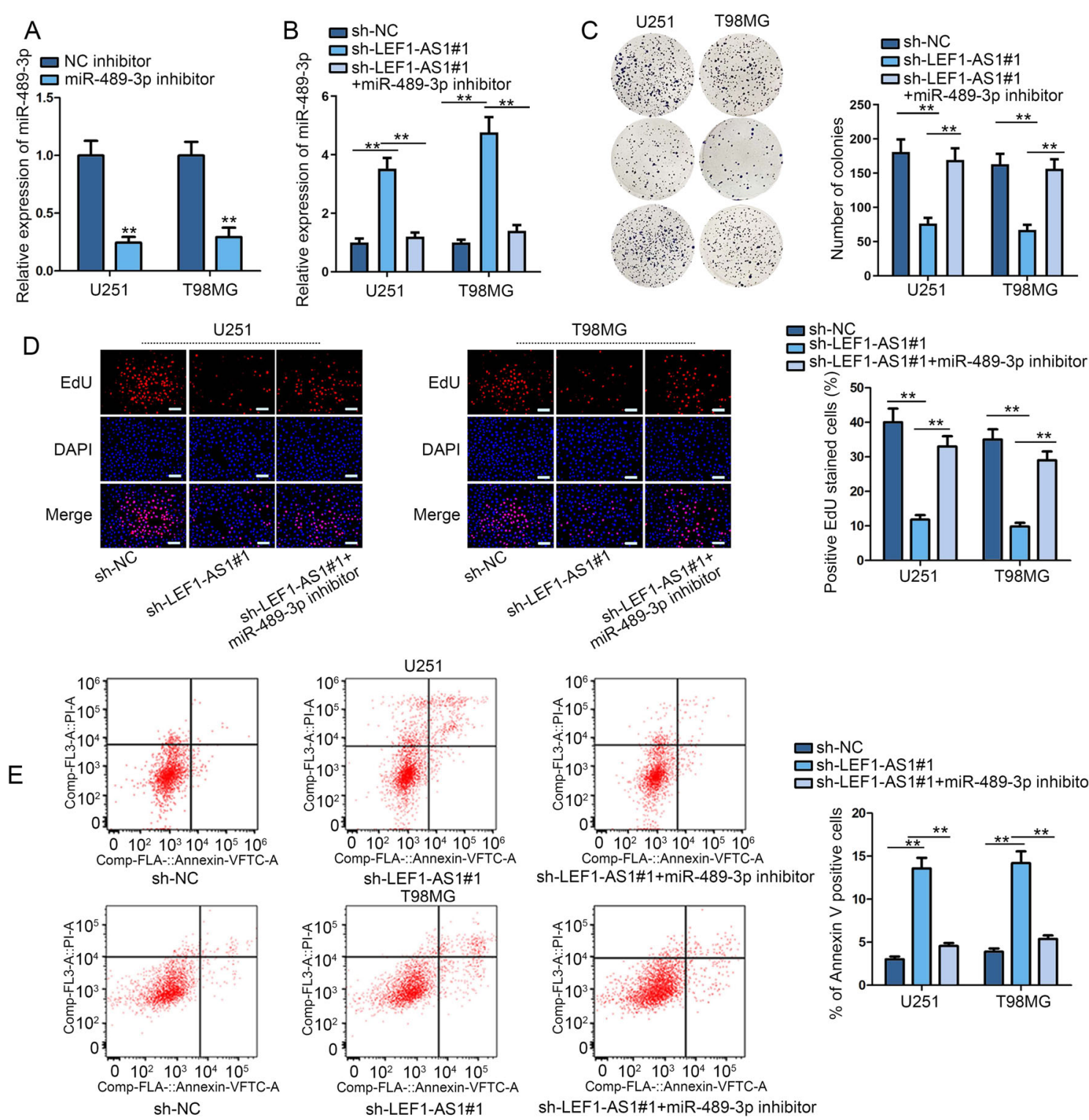
sh-LEF1-AS1\#1+miR-489-3p inhibitor

$\square$ sh-NC

口Sh-LEF1-AS1\#1

sh-LEF1-AS1\#1+miR-489-3p inhibitor

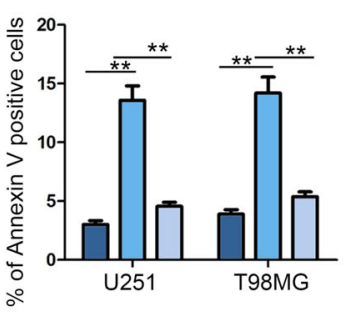

F

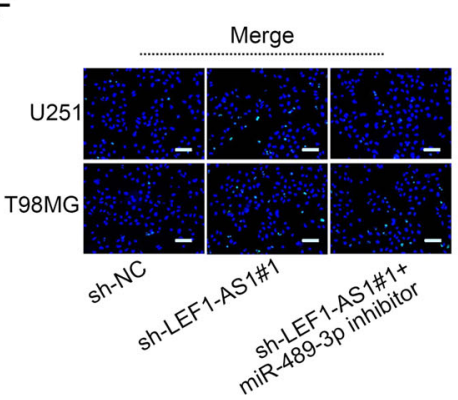

$\square$ sh-NC

$\square$ sh-LEF1-AS1\#1

$\square$ sh-LEF1-AS1\#1+miR-489-3p inhibitor

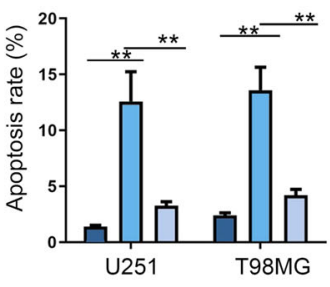

G

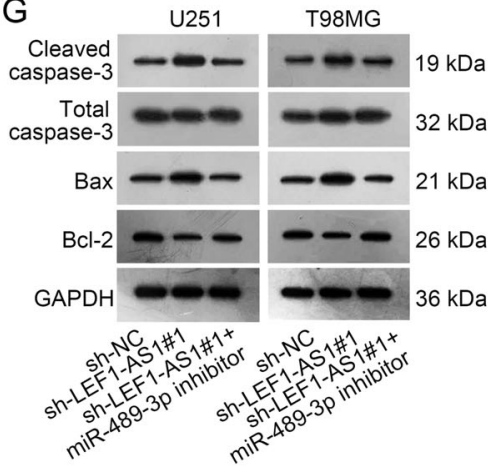

Fig. 3 MiR-498-3p depletion reversed the effects of LEF1-AS1 knockdown on glioma cells. $\mathbf{a}, \mathbf{b}$ MiR-489-3p knockdown efficiency and its expression in differently transfected groups were examined by qRT-PCR in U251 and T98MG cells. c, d Colony formation and EdU (scale bar $=$ $100 \mu \mathrm{m}$ ) assays measured the proliferation ability of glioma cells transfected with sh-NC, sh-LEF1-AS1\#1 or sh-LEF1-AS1\#1+miR-498-3p inhibitor. e, f Flow cytometry analysis and TUNEL (scale bar $=100 \mu \mathrm{m}$ ) assay evaluated cell apoptosis ability under above conditions. $\mathbf{g}$ Western blot was performed to measure the expression of apoptosis-related proteins in indicated cells. ${ }^{*} P<0.01$. 

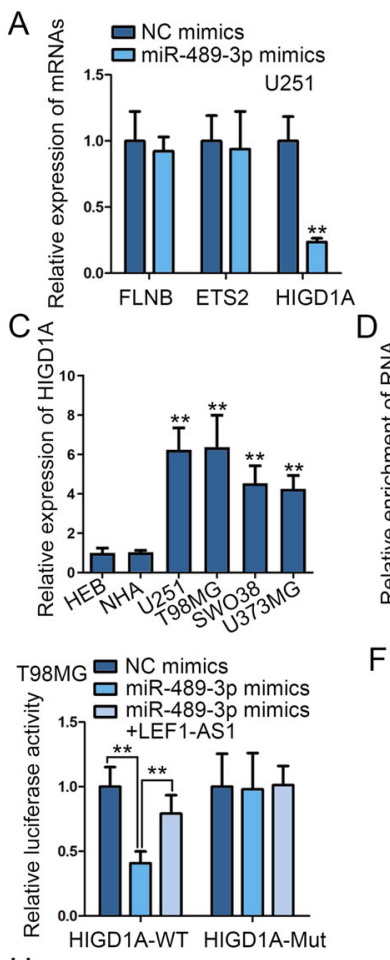

$\mathrm{H}$

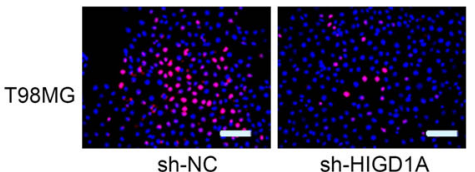

U251
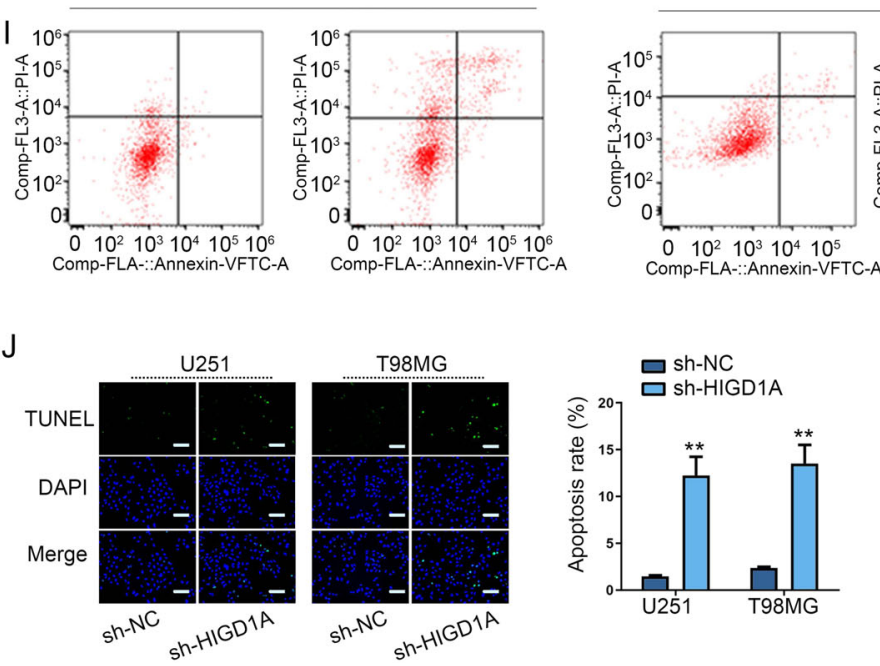

B

HIGD1A-WT: 5 ' ccuguuugcugcagaAUGUCAg 3 || | ||

miR-489-3p : 3' cgacggcauauacacUACAGUg 5'

HIGD1A-Mut: $\quad$ ' ccuguuugcugcagaUACAGUg 3
T98MG

E U251 $\square$ miR-489-3p mimics त miR-489-3p mimics
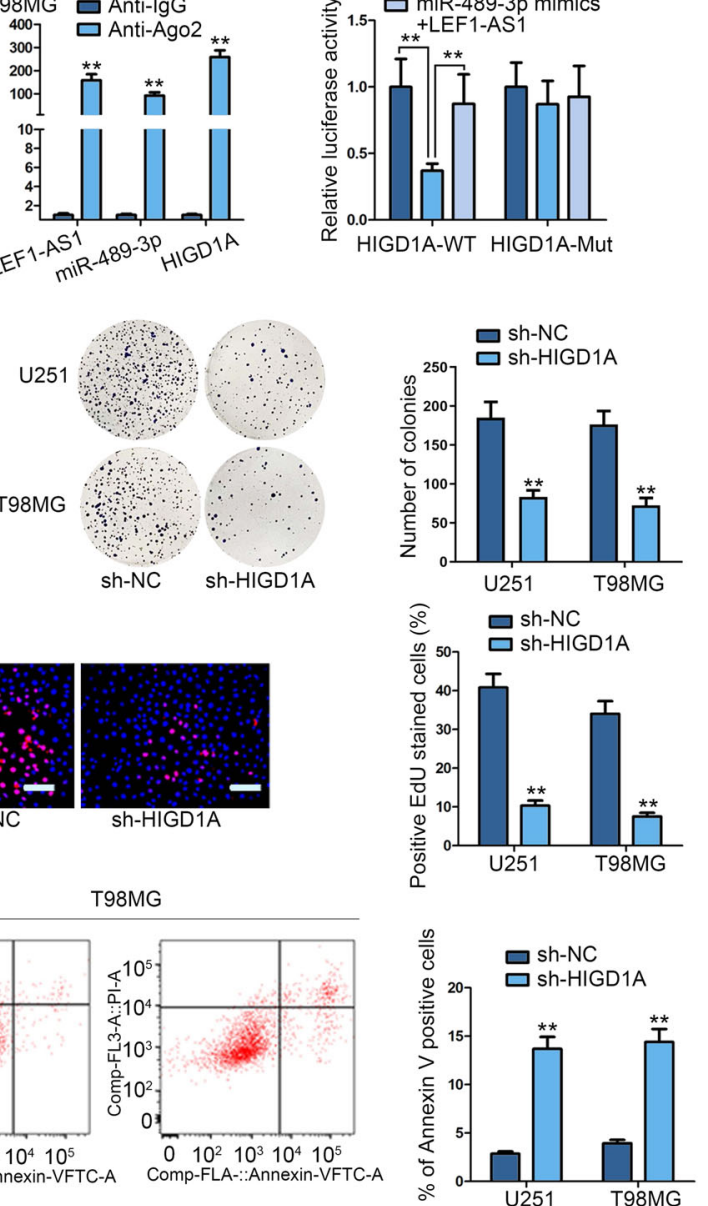

$\mathrm{K}$

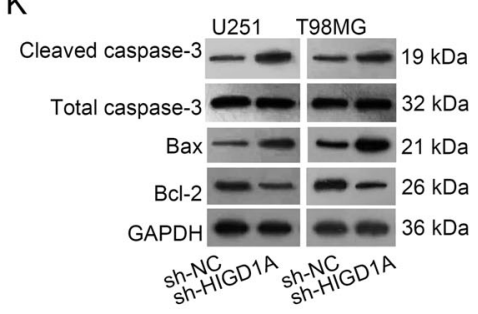

Fig. 4 HIGD1A silence curbed the process of glioma cells. a The expression of FLNB, ETS2 and HIGD1A was measured by qRT-PCR when overexpressing miR-489-3p. b Bioinformatics (starBase) predicted the binding sites between miR-489-3p and HIGD1A. c HIGD1A expression was assessed by qRT-PCR in glioma cells and control cells. $\mathbf{d}$ RIP assay testified the enrichment of LEF1-AS1, miR-489-3p and HIGD1A in Anti-Ago2 or AntiIgG group. e Luciferase reporter assay examined the luciferase activity of HIGD1A-WT/Mut in miR-489-3p mimics, miR-489-3p mimics+LEF1-AS1 or NC mimics group (f) HIGD1A knockdown efficiency was measured by qRT-PCR in U251 and T98MG cells. $\mathbf{g}-\mathbf{j}$ The influence of sh-HIGD1A on glioma cell proliferation and apoptosis was measured by colony formation assay, EdU assay (scale bar $=100 \mu \mathrm{m}$ ), flow cytometry analysis and TUNEL assay $($ scale bar $=100 \mu \mathrm{m})$. $(\mathbf{k})$ Western blot was conducted to evaluate the expression apoptosis-related proteins when knocking down HIGD1A. ${ }^{* *} P<0.01$. 


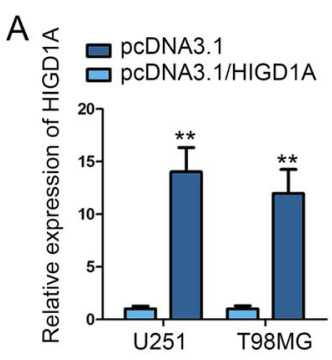

C

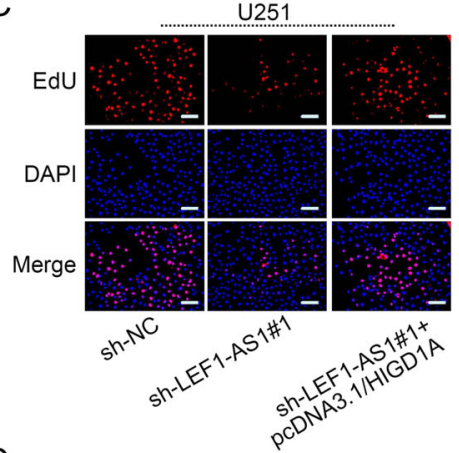

B

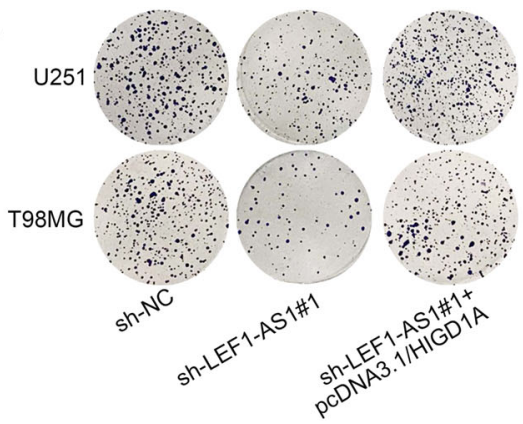

T98MG

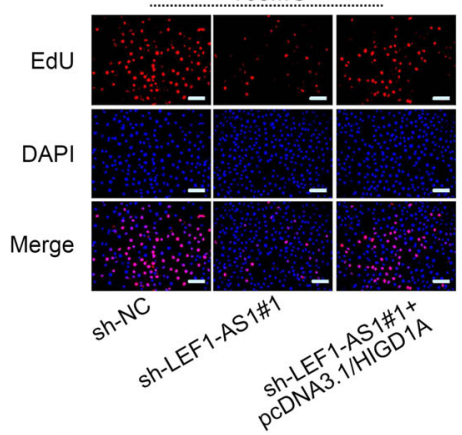

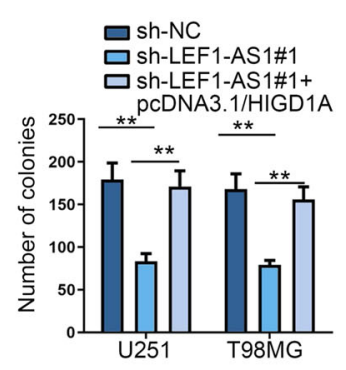

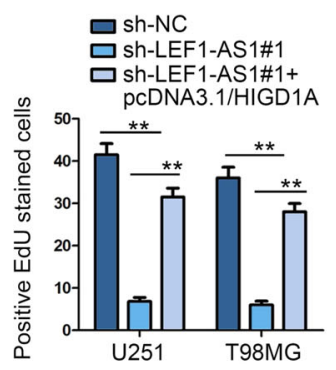

D
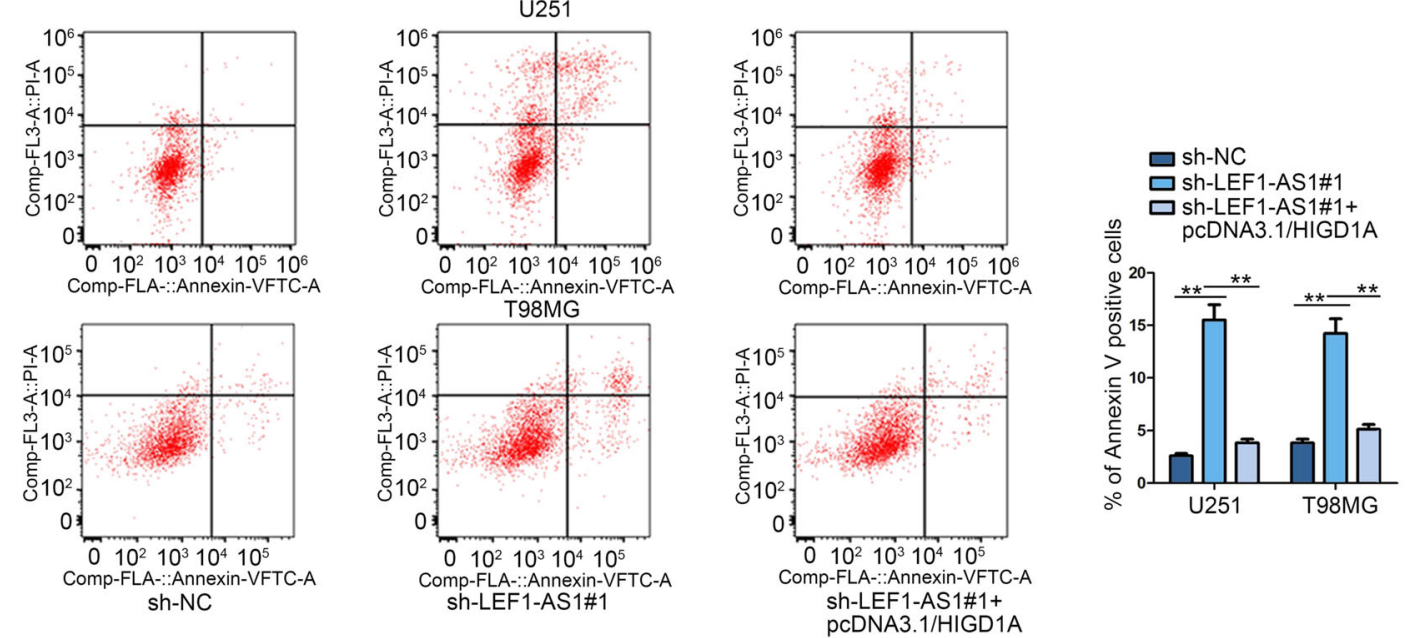

E

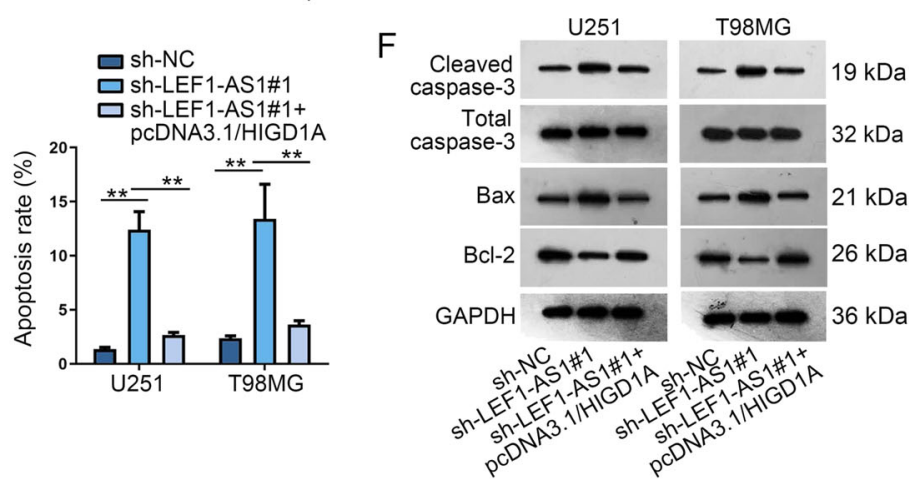

Fig. 5 LEF1-AS1 fostered the malignant course in glioma via enhancing HIGD1A expression. a Overexpression efficiency of HIGD1A waS validated by qRT-PCR. $\mathbf{b}$ - $\mathbf{f}$ The influence of sh-LEF1-AS1\#1 or sh-LEF1-AS1\#1 + pCDNA3.1/HIGD1A on glioma cell proliferation and apoptosis was assessed by colony formation assay, EdU assay (scale bar $=100 \mu \mathrm{m}$ ), flow cytometry analysis, TUNEL assay (scale bar $=100 \mu \mathrm{m})$ and western blot analysis. ${ }^{* *} P<0.01$. 


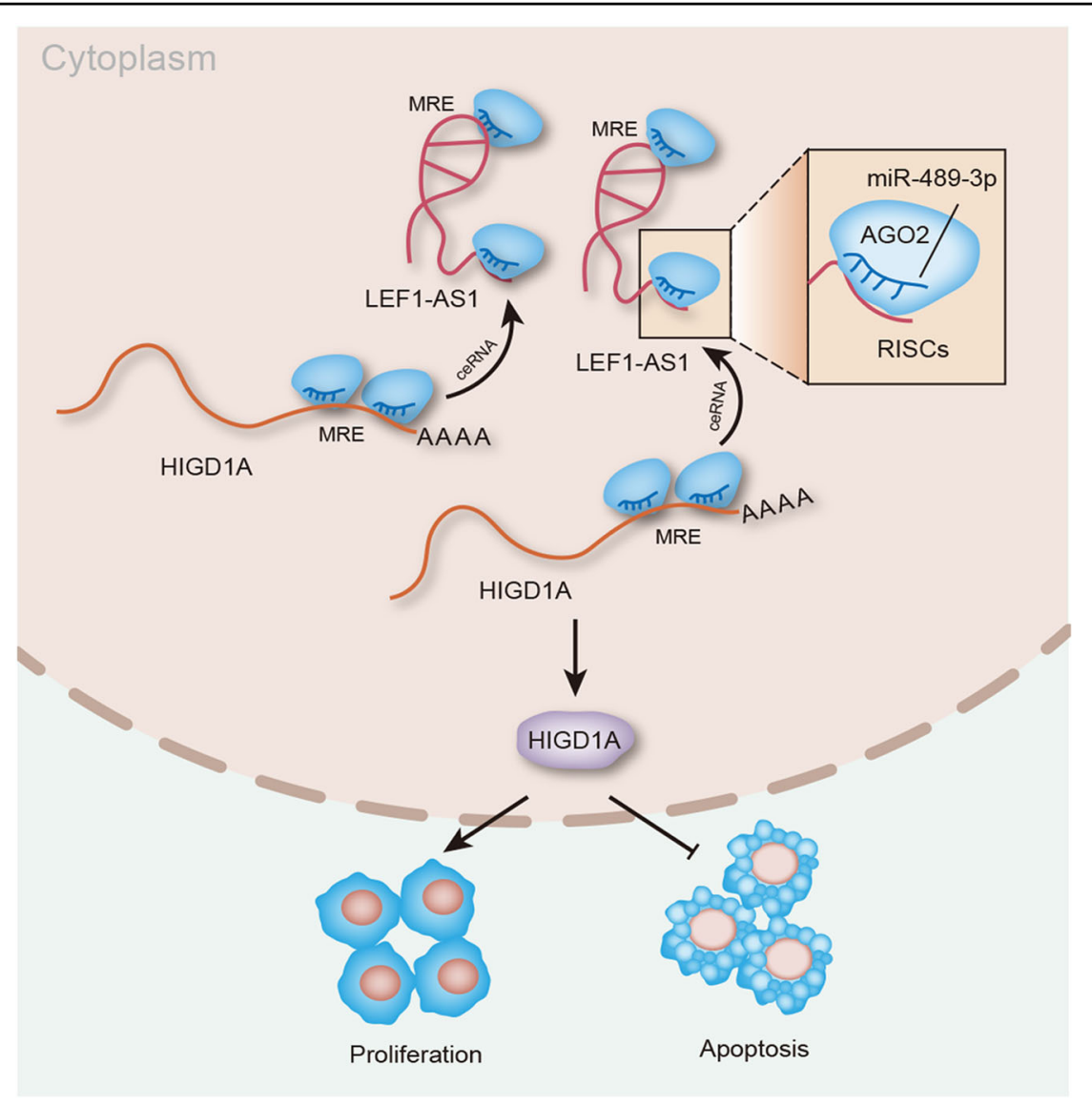

Fig. 6 Graphical diagram. LEF1-AS1/miR-489-3p/HIGD1A axis in glioma.

LEF1-AS1 silence-mediated suppressive function on cell proliferation (Fig. 5b, c). In terms of glioma cell apoptosis, the accelerating effects of LEF1-AS1 depletion on apoptosis was neutralized by augmented HIGD1A, suggested by flow cytometry analysis and TUNEL assay (Fig. 5d, e). Western blot pictured that the encouraging influence of LEF1-AS1 knockdown on cleaved caspase-3 and Bax expressions and suppressing influence of that on Bcl-2 expression could be counterbalanced by HIGD1A overexpression (Figs. $5 \mathrm{f}$ and S3c). In general, LEF1-AS1 facilitated glioma cell proliferation and inhibited cell apoptosis through function as a ceRNA to target miR-489$3 p$ and thereby upregulated HIGD1A expression (Fig. 6).

\section{Discussion}

In the late decades, the crucial influence of lncRNAs in glioma has been well-accepted such as its modulation on metastasis, chemoresistance and growth ${ }^{17,18}$. For example, H19 was described as an oncogene in glioma via inducing angiogenesis through repressing miR-29a ${ }^{19}$. LncRNA TUNAR was disclosed to suppress metastasis and proliferation in glioma via modulating miR-200a and $\operatorname{Rac}^{20}$. MALAT1 regulated cell infiltration and treatment resistance in glioblastoma multiforme ${ }^{21}$. These findings provide insights into researching pathogenesis of glioma. Preliminary works reported that LEF1-AS1 worked as a potent oncogenic player in oral squamous cell carcinoma $(\mathrm{OSCC})^{22}$. Noticeably, we examined that LEF1-AS1 was an upregulated lncRNA in glioma tissues and found that it was associated with short survival rate of glioma patients, which interested us to explore its role in glioma. LEF1-AS1 knockdown suppressed glioma cell proliferation in vitro and tumor growth in vivo, indicating LEF1-AS1 as a tumor facilitator in glioma.

Considering the cytoplasmic abundance of LEF1-AS1 in glioma cells and the vital role of cytoplasmic lncRNAs in ceRNA pattern, we unveiled the underlying interplay between LEF1-AS1 and miR-489-3p. Through experimental works, it was certified that miR-489-3p negatively regulated by LEF1-AS1, consistent with previous research findings ${ }^{23,24}$. In addition, miR-489-3p was lowly expressed in glioma cells. Furthermore, it was found that miR-489$3 p$ depletion could restore the influences of LEF1-AS1 silence on glioma cell proliferation and apoptosis. Besides, miR-489-3p was identified as a tumor suppressor in renal cell carcinoma ${ }^{25,26}$ and bladder cancer ${ }^{27}$. Taken together, 
our study first shed new light on the relationship between LEF1-AS1 and miR-489-3p in glioma.

The regulatory effects of HIGD1A have been wellcharacterized in cellular survival and apoptosis in human carcinomas ${ }^{28,29}$. In this study, we uncovered that HIGD1A was the downstream target of miR-489-3p. In addition, HIGD1A was tested to be significantly upregulated in glioma cells. Depletion of HIGD1A led to decreased cell proliferation and activated apoptosis in glioma. Additionally, LEF1-AS1 knockdown-mediated function in the behaviors of glioma cells was offset by overexpressing HIGD1A.

In a word, our work elucidated that LEF1-AS1 interacts with miR-489-3p to relieve the suppressing effect of miR489-3p on HIGD1A mRNA so as to increase the expression of HIGD1A, thereby promoting cell proliferation and inhibiting apoptosis in glioma, evidencing that LEF1-AS1 might serve as a potential therapeutic target for glioma patients.

\section{Acknowledgements}

All supports from participants in this research were undeniable.

\section{Conflict of interest}

The authors declare that they have no conflict of interest.

\section{Publisher's note}

Springer Nature remains neutral with regard to jurisdictional claims in published maps and institutional affiliations.

Supplementary Information accompanies this paper at (https://doi.org/ 10.1038/s41419-020-02823-0).

Received: 28 October 2019 Revised: 17 June 2020 Accepted: 18 June 2020 Published online: 11 August 2020

\section{References}

1. Nayak, L. \& Reardon, D. A. High-grade gliomas. Contin. (Minneap., Minn.) 23, 1548-1563 (2017)

2. Allen, D. H. \& Loughan, A. R. Impact of cognitive impairment in patients with gliomas. Semin. Oncol. Nurs. 34, 528-546 (2018).

3. Chen, Y. et al. Long non-coding RNA HCG11 modulates glioma progression through cooperating with miR-496/CPEB3 axis. Cell Prolif. 52, e12615 (2019).

4. Reifenberger, G., Wirsching, H. G., Knobbe-Thomsen, C. B. \& Weller, M. Advances in the molecular genetics of gliomas-implications for classification and therapy. Nat. Rev. Clin. Oncol. 14, 434-452 (2017).

5. Tan, S. K. et al. Serum long noncoding RNA HOTAIR as a novel diagnostic and prognostic biomarker in glioblastoma multiforme. Mol. cancer 17, 74 (2018).

6. Zhou, K. et al. Knockdown of long non-coding RNA NEAT1 inhibits glioma cell migration and invasion via modulation of SOX2 targeted by miR-132. Mol. cancer 17, 105 (2018).

7. Han, M. et al. Interfering with long non-coding RNA MIR22HG processing inhibits glioblastoma progression through suppression of Wnt/beta-catenin signalling. Brain: J. Neurol., https://doi.org/10.1093/brain/awz406 (2019).
8. Liu, D. C. et al. Long noncoding RNA LEF1-AS1 silencing suppresses the initiation and development of prostate cancer by acting as a molecular sponge of miR-330-5p via LEF1 repression. J. Cell. Physiol. 234, 12727-12744 (2019).

9. Congrains-Castillo, A., Niemann, F. S., Santos Duarte, A. S. \& Olalla-Saad, S. T. LEF1-AS1, long non-coding RNA, inhibits proliferation in myeloid malignancy. J. Cell. Mol. Med. 23, 3021-3025 (2019).

10. $\mathrm{Xu}, \mathrm{M}$. et al. The long noncoding RNA SNHG1 regulates colorectal cancer cell growth through interactions with EZH2 and miR-154-5p. Mol. Cancer 17, 141 (2018).

11. $\mathrm{Yu}, \mathrm{M}$. et al. Linc00152 promotes malignant progression of glioma stem cells by regulating miR-103a-3p/FEZF1/CDC25A pathway. Mol. Cancer 16, 110 (2017).

12. Pandolfini, L. et al. METTL1 promotes let-7 MicroRNA processing via $m 7 G$ methylation. Mol. cell 74, 1278-1290.e1279 (2019).

13. Bhaskaran, $\mathrm{V}$. et al. The functional synergism of microRNA clustering provides therapeutically relevant epigenetic interference in glioblastoma. Nat. Commun. 10, 442 (2019).

14. Wu, X. et al. Blocking MIR155HG/miR-155 axis inhibits mesenchymal transition in glioma. Neuro-Oncol. 19, 1195-1205 (2017).

15. Wang, R. et al. EIF4A3-induced circular RNA MMP9 (circMMP9) acts as a sponge of miR-124 and promotes glioblastoma multiforme cell tumorigenesis. Mol. Cancer 17, 166 (2018).

16. Li, S. et al. miR-423-5p contributes to a malignant phenotype and temozolomide chemoresistance in glioblastomas. Neuro-Oncol. 19, $55-65$ (2017).

17. $\mathrm{Hu}, \mathrm{Q}$. et al. H19 Functions as a competing endogenous RNA to regulate EMT by sponging miR-130a-3p in glioma. Cell. Physiol. Biochem.: Int. J. Exp. Cell. Physiol. Biochem. Pharmacol. 50, 233-245 (2018).

18. Xu, D. et al. Long non-coding RNA ENST01108 promotes carcinogenesis of glioma by acting as a molecular sponge to modulate miR-489. Biomed. Pharmacother. $=$ Biomed. Pharmacotherapie 100, 20-28 (2018).

19. Jia, P. et al. Long non-coding RNA H19 regulates glioma angiogenesis and the biological behavior of glioma-associated endothelial cells by inhibiting microRNA-29a. Cancer Lett. 381, 359-369 (2016).

20. Dai, J. et al. Long noncoding RNA TUNAR represses growth, migration, and invasion of human glioma cells through regulating miR-200a and Rac1. Oncol. Res. 27, 107-115 (2018),

21. Kim, S. S. et al. Targeted nanocomplex carrying siRNA against MALAT1 sensitizes glioblastoma to temozolomide. Nucleic Acids Res. 46, 1424-1440 (2018).

22. Zhang, C. et al. Knockdown of IncRNA LEF1-AS1 inhibited the progression of oral squamous cell carcinoma (OSCC) via Hippo signaling pathway. Cancer Biol. Ther. 20, 1213-1222 (2019).

23. $\mathrm{Li}, \mathrm{H}$. et al. Long noncoding RNA NORAD, a novel competing endogenous RNA, enhances the hypoxia-induced epithelial-mesenchymal transition to promote metastasis in pancreatic cancer. Mol. Cancer 16, 169 (2017).

24. Wang, Y. et al. Long noncoding RNA DANCR, working as a competitive endogenous RNA, promotes ROCK1-mediated proliferation and metastasis via decoying of miR-335-5p and miR-1972 in osteosarcoma. Mol. Cancer 17, 89 (2018).

25. Chen, L. et al. Upregulation of miR-489-3p and miR-630 inhibits oxaliplatin uptake in renal cell carcinoma by targeting OCT2. Acta Pharmaceutica Sin. B $\mathbf{9}$, 1008-1020 (2019).

26. Liu, Q., Yang, G. \& Qian, Y. Loss of MicroRNA-489-3p promotes osteosarcoma metastasis by activating PAX3-MET pathway. Mol. Carcinogenesis 56, 1312-1321 (2017)

27. $L i$, J. et al. miR-489 suppresses proliferation and invasion of human bladder cancer cells. Oncol. Res. 24, 391-398 (2016).

28. Ameri, K. \& Maltepe, E. HIGD1A-mediated dormancy and tumor survival. Mol. Cell. Oncol. 2, e1030537 (2015).

29. Ameri, K. et al. HIGD1A regulates oxygen consumption, ROS production, and AMPK activity during glucose deprivation to modulate cell survival and tumor growth. Cell Rep. 10, 891-899 (2015). 